\title{
Van den Bosch syndrome
}

INSERM

\section{Source}

INSERM. (1999). Orphanet: an online rare disease and orphan drug data base. Van den Bosch syndrome. ORPHA:3417

Van den Bosch syndrome is characterized by intellectual deficit, choroideremia, acrokeratosis verruciformis, anhidrosis, and skeletal deformities. It has been observed in a single kindred. The syndrome is transmitted as an X-linked recessive trait and may be caused by a small X-chromosome deletion. 